\title{
Organic Photoredox-Catalyzed Cycloadditions Under Single-Chain Polymer Confinement
}

\author{
Jacob J. Piane, Lauren E. Chamberlain, Lucas T. Alameda, Ashley C. Hoover, and Elizabeth Elacqua* \\ Department of Chemistry, The Pennsylvania State University, University Park PA, 16802, USA
}

\begin{abstract}
Cooperative catalysis enables synthetic transformations that are not feasible using monocatalytic systems. Such reactions are often diffusion controlled and require multiple catalyst interactions at high dilution. We developed a confined dualcatalytic polymer nanoreactor that enforces catalyst co-localization to enhance reactivity in a fully-homogeneous system. The photocatalyzed-dimerization of substituted styrenes is disclosed using confined-single-chain polymers bearing triarylpyrylium-based pendants, with pyrene as an electron relay catalyst. Enhanced reactivity with low catalyst loadings was observed compared to monocatalytic polymers with small-molecule additives. Our approach realizes a dual-catalytic single-chain polymer that provides enhanced reactivity under confinement, presenting a further approach for diffusion-limited-photoredox catalysis.
\end{abstract}

Compartmentalization is one of Nature's design principles: enzymes are shielded from incompatible environments and partitioned such that cooperative functions like catalysis are optimized. Confined-space effects in catalysis have also been reported with organic molecules and porous materials (e.g., cucurbit[n]urils, metal-organic frameworks (MOFs), and metalorganic cages (MOCs)). Systems like MOFs or MOCs provide distinct benefits, including periodic arrangements of transition metal catalysts that facilitate increased local concentrations of reactive species, ${ }^{1}$ thus, accelerating the rate of organic reactions. Localized rate enhancement is also observed in molecular systems, such as the hydrogen-binding Rebek's softball, in which reactive species diffused toward a catalytic center allowing for a 200 -fold rate enhancement. ${ }^{2}$ In close analogy to Nature, these supramolecular frameworks also aid in stabilization of different conformations. Raymond's $\mathrm{M}_{4} \mathrm{~L}_{6}$ cluster, ${ }^{3}$ for example, has a unique interior microenvironment that lowers entropic barriers to reactivity, while enthalpically favoring compact transition states that are not observable in bulk solution.

Recently, sunlight-enabled-photocatalysis has emerged as a pillar for synthesis, ${ }^{4-6}$ particularly for $\mathrm{C}-\mathrm{C}$ and $\mathrm{C}-\mathrm{N}$ bond construction while exploiting mild reaction conditions. Commonly-employed photocatalysts (PCs) are based upon iridium or ruthenium metal complexes and organic dyes that feature long excited-state lifetimes, high redox potentials, and strong visible absorption. Dual photoredox catalysis enables challenging transformations that cannot be achieved with either catalyst alone. ${ }^{7}$ The merger of photoredox with transition metal catalysis has achieved multiple $\mathrm{C}-\mathrm{H}$ activation reactions with $\mathrm{Pd},{ }^{8}$ as well as trifluoromethylation, ${ }^{9}$ difunctionalization, ${ }^{10}$ and other strategies with copper co-catalysts. ${ }^{11}$ Much interrogation into dual photoredox/Ni catalysis has unleashed potent $\mathrm{sp}^{3}-\mathrm{sp}^{2}$ cross-coupling methods from the groups of MacMillan, ${ }^{12,}{ }^{13}$ Doyle, ${ }^{14}$ and Molander, ${ }^{15}, 16$ including decarboxylative and organoboron couplings. ${ }^{17}$ Additional strategies by Yoon and coworkers have interrogated Bronsted and Lewis acids as cocatalysts for metallaphotoredox-mediated [2+2] cycloaddition reactions, leading to high control over product chirality. ${ }^{18-20}$ Further, Nicewicz has pioneered a photoredox/electron relay co-catalysis system for [2+2] dimerizations to realize natural lignan-based cyclobutanes. ${ }^{21}$

The efficiency of co-catalytically-powered reactions relies on proximal catalyst locations, a bottleneck difficult to control with the degrees of freedom often afforded in solution-phase chemistry. In addition, challenges remain in optimization, as expensive PCs can be loaded at amounts exceeding maximum solubility. ${ }^{22}$ Strategies to circumvent these limitations in heterogeneous photoredox catalysis have been realized using MOFs and quantum dots (QDs) by the groups of Lin and Weix, ${ }^{23}$ respectively. While the MOF confines Ir- and Ni-catalytic components within $0.6 \mathrm{~nm}$ of each other ${ }^{24}$ and facilitates electron and radical transfers between them that allows for efficient turnover, the QDs were highly effective at extremely low loadings.

Considering the successful heterogeneous dual catalysis in MOFs, we hypothesized that single-chain polymer nanoparticles (SCNPs) ${ }^{25}$ could provide a versatile platform to drive homogeneous dual catalysis within nanoreactors. The SCNP would provide several advantages, including controlled catalyst loadings, solubility, and well-defined crosslinking that enables co-localization of cooperative catalysts. Herein, we disclose a triarylpyrylium (TPT)-based polymer that functions as an organic single-electron oxidant/electron relay nanoreactor. Our design features a styrylpyrene (SP) monomer that acts as both a covalent crosslink ${ }^{26}$ to generate the confined environment, but also as a functioning electron relay (ER) for photoredox-catalyzed stereospecific [2+2] cycloadditions. Our TPT-SP-based nanoreactor operates in low loadings of both the photocatalyst and ER ( 1 mol \% TPT, $0.67 \mathrm{~mol} \%$ ER), and demonstrates enhanced reactivity in comparison to monocatalytic polymer analogues. We attribute these results to efficient colocalization of the PC and ER, owing to confinement.

Our motivation lies in developing versatile and efficient homogeneous catalysts. In studies to realize co-catalytically-active polymers for sustainable chemistry, we noted that SCNPs 
whose folding featured metal crosslinks ${ }^{27}$ had demonstrated reasonable catalytic activity in reactions such as oxidations and cross-couplings. ${ }^{25}$ Further reports by Zimmerman identified a single-chain polymeric 'Clickase' that accelerates copper-click reactions. ${ }^{28}$ Given the success of these monocatalytic systems, combined with recent reports of enhanced reactivity, ${ }^{28}$ we intuited that a SCNP comprising another catalyst could promote accelerated reactions under confinement if the crosslink could additionally act as an organocatalyst. Recognizing the potential of pyrene to act as an ER catalyst, we designed SCNPs bearing strongly-oxidizing TPT and pyrene to study the effects of confining two cooperative catalysts in close proximity. Photocatalyzed-[2+2] cycloadditions are reported with electron-rich styrenes $^{29}, 30$ When strongly oxidizing photocatalysts are employed, cycloreversion of the resulting cycloadduct can predominate and shift the equilibrium of this reaction towards the starting alkene. The addition of a polyaromatic electron relay catalyst circumvents oxidative cycloreversion by acting as the active oxidant with a lower potential that is not sufficient to oxidize the cyclized product. ${ }^{21}$ Specifically, the potent photooxidant first oxidizes the ER catalyst, which subsequently oxidizes the substrate. Honing in on the interaction between the two catalysts, we hypothesized that confining the photoredox catalyst and ER within the same polymer backbone would enhance the kinetics of single-electron transfer.

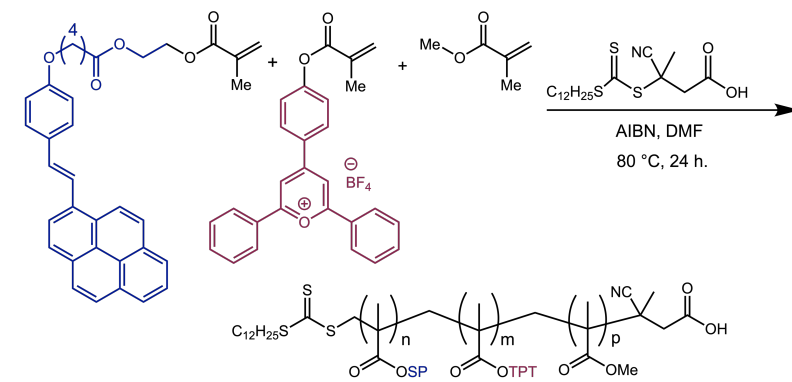

\begin{tabular}{ccccccc}
\hline Polymer & $\begin{array}{c}\text { Target Ratio } \\
\text { [MMA]:[TPT]:[SP] }\end{array}$ & $\begin{array}{c}\% \text { TPT } \\
\text { by NMR }\end{array}$ & $\begin{array}{c}\% \text { SP } \\
\text { by NMR }\end{array}$ & Mn & $\begin{array}{c}\text { Mw } \\
\text { (kDa) }\end{array}$ & D \\
\hline TPT-SP-polymer & {$[90]$ [5]:[5] } & 6 & 4 & 12.0 & 17.0 & 1.45 \\
TPT-SCNP & N/A & 6 & 4 & 14.0 & 19.0 & 1.35 \\
SP-co-MMA & N/A & N/A & 0.68 & 18.3 & 21.5 & 1.02 \\
SP-SCNP & N/A & N/A & 0.68 & 19.3 & 22.8 & 1.18 \\
TPT-co-MMA & N/A & 1.83 & N/A & 15.2 & 17.9 & 1.17
\end{tabular}

Scheme 1: Schematic depicting TPT-SP-polymer synthesis and individual polymer characterization information.

Triarylpyrylium salts are easily prepared with a diverse range of functionalities at the aryl positions, providing access to photocatalysts with varying electronic properties. This facilitates tuning excited-state redox potentials. Methacrylate-derived pyrylium monomers were prepared according to previous reports. ${ }^{31}$ Methyl methacrylate (MMA) was selected as the backbone in order to maintain solubility in common organic solvents. With the desired monomers in hand, statistical copolymers comprising TPT-methacrylate, SP-methacrylate, and MMA were prepared with targeted incorporation of 90:5:5 [MMA]:[TPT]:[SP]. Polymers were synthesized using reversible-addition fragmentation chain-transfer (RAFT) polymerization (Scheme 1). ${ }^{1} \mathrm{H}-\mathrm{NMR}$ spectroscopic characterization confirmed a $6 \%$ incorporation of TPT and $4 \%$ incorporation of SP. The polymer molecular weight was determined to be $12 \mathrm{kDa}$ through size exclusion chromatography (SEC). SEC also confirmed that the polymers were well-defined $(\bigoplus=1.45)$ (Figure $1)$.
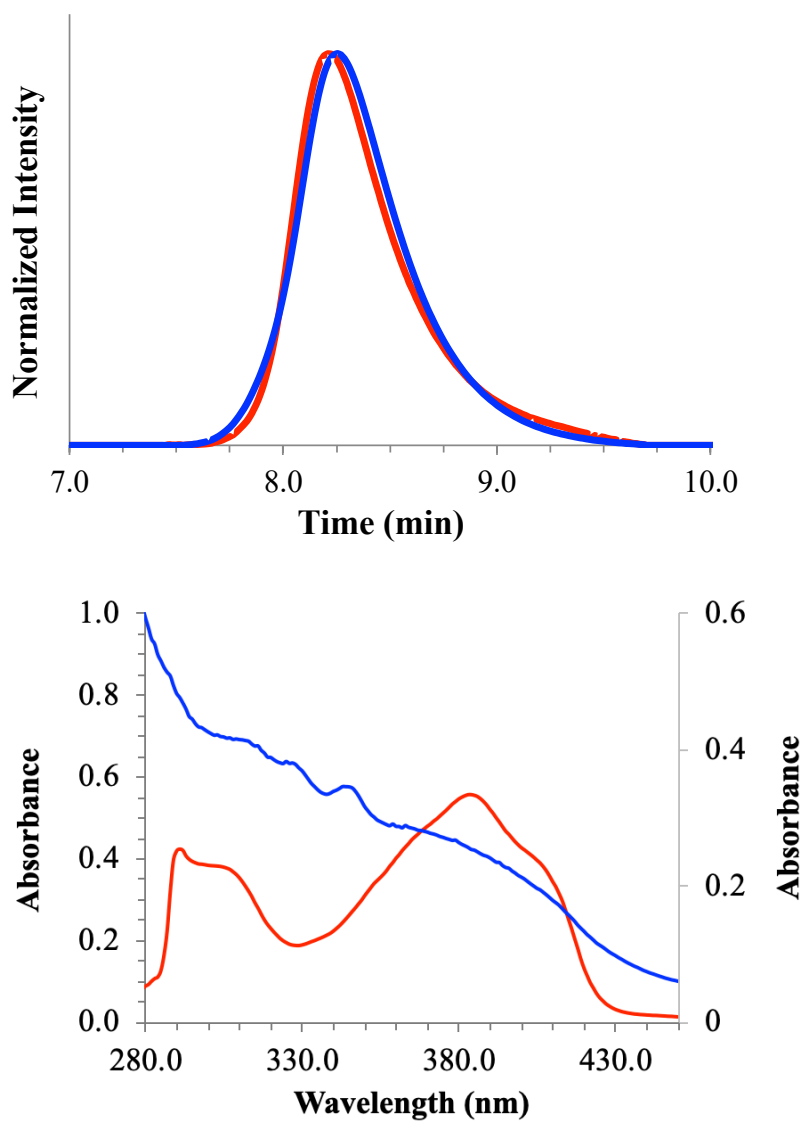

Figure 1: (top) SEC overlay and (bottom) UV/Vis spectral overlay of TPT-SP-polymer (red) and SCNP (blue, secondary axis).

Cross-linking of styrylpyrene residues was achieved by irradiation of linear polymer solutions in $\mathrm{MeCN}(10 \mathrm{mg} / \mathrm{mL})$ with a white compact fluorescent lamp. Dimerization of styrylpyrene and nanoparticle formation was characterized by UV/Vis spectroscopy, wherein disappearance of the characteristic styrenyl absorbance $(\lambda=391 \mathrm{~nm})$ and concomitant appearance of cyclobutane features at $\lambda=333 \mathrm{~nm}$ and $352 \mathrm{~nm}$ were observed (Figure 1). SEC confirmed primarily intramolecular cross-linking with a small increase in apparent molecular weight to $14 \mathrm{kDa}$ and dispersity of 1.35 (Figure 1).

Trans-anethole was selected as a model system to study the $[2+2]$ cyclodimerization using the SCNP (Figure 2), given prior reports of TPT-catalyzed single-electron oxidant-electron relay photocatalysis in the cyclodimerization of anethole. ${ }^{21}$ In a typical experiment, the styrenic small molecule was added to a solution of the SCNP (0.0004 mmol loading, which corresponds to $1.0 \mathrm{~mol} \%$ TPT) in MeCN, and was irradiated with $427 \mathrm{~nm}$ Kessil LEDs at 25\% light intensity (approximate light-to-vial distances of $\sim 7.5 \mathrm{~cm}$ ), with conversion monitored using ${ }^{1} \mathrm{H}$ NMR spectroscopy. After evaluating several solvents, MeCN was found to provide the highest yield (See SI). This was attributed to increased solubility of the polymer and high dielectric constant. When using the confined TPT-SCNP photocatalyst bearing the pyrene-based ER, a $66 \%$ yield of the dimerized product was observed within 24 hours. Increasing the concentration to $0.4 \mathrm{M}$ gave moderate improvements of yield $(71 \%$, Table S-1), while further changes adversely affected product formation. Altering the photocatalyst concentration and increasing light intensity decreased yield. The pyrylium family of photoredox catalysts is prone to photobleaching and 
dimerization of the pyranyl radical; ${ }^{32,33}$ accordingly, longer reaction times did not yield more product, likely because the amount of active pyrylium in solution decreases over time. Reactions conducted without any TPT-SCNP or in the absence of light yielded no cycloadduct.
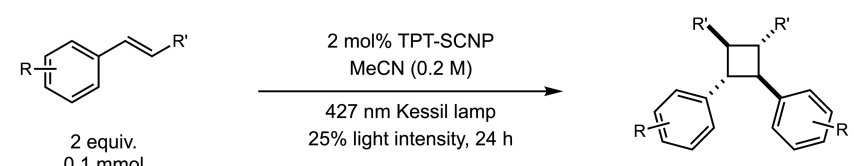

$0.1 \mathrm{mmol}$

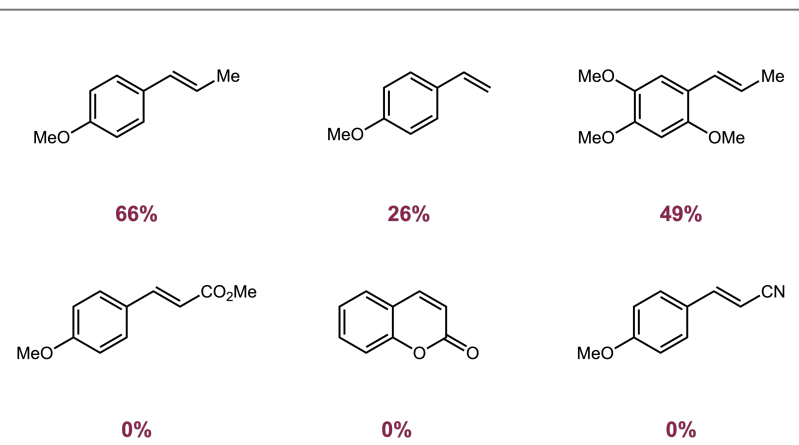

Figure 2: [2+2] Cycloadditions of styrenyl derivatives through TPT-SCNP-catalyzed photoinduced-electron transfer.

Typically, visible-light-organocatalyzed [2+2] cyclodimerizations proceed in a sluggish manner, often over $48+$ hours, and reported TPT-catalyzed cycloadditions similarly plateau in conversion over days. Thus, we sought to further investigate the SCNP system and elucidate the effect of confinement. Given the optimized results suggested that the photocatalyzed cycloaddition proceeded with ease, control experiments were conducted using monocatalytic polymers and compared with the dual catalytic SCNPs (Table 1). We synthesized copolymers of MMA with TPT-methacrylate or SP-methacrylate. Both polymers were prepared by RAFT polymerization using the same procedure as for the target co-catalytic polymer. Proton NMR spectroscopy indicated $1.83 \%$ incorporation of TPT and $0.68 \%$ incorporation of SP in TPT-co-MMA and SP-co-MMA, respectively (Scheme 1). The molecular weight of the TPT copolymer was determined to be $18 \mathrm{kDa}$ using SEC in THF, with a dispersity of 1.17 . The molecular weight of the SP copolymer was determined to be $21.5 \mathrm{kDa}$, with a dispersity of 1.02 . Similar to the co-catalytic SCNP, the SP-co-MMA monomer was crosslinked to mimic a confined network, denoted SP-SCNP.

With the monocatalytic polymers in hand, we attempted to catalyze the [2+2] cycloaddition of trans-anethole with TPTco-MMA in the absence of the ER. Using the same optimized conditions resulted in $2 \%$ of the desired product (and recovering mostly starting material) after 24 hours, suggesting either the presence of the ER or the confined interior is critical. Further, irradiation of trans-anethole with TPT-co-MMA as the PC in the presence of pyrene as a small-molecule additive, similarly resulted in trace amounts of cyclobutane adduct. These results suggested confinement was a significant design element and led to accelerated rates and higher conversion when using the SCNP. We, thus investigated the possibility that SP-SCNP could similarly function as a nanoreactor, providing small molecule TPT and the reactant could efficiently diffuse toward the ER for co-catalysis. This reaction afforded the desired product in $10 \%$ yield after 24 hours, while the same reaction in the absence of TPT gave none of the desired product. Further, when the TPT-co-MMA polymer and SP-SCNP polymers were used together as co-catalysts, trace amounts of product were observed. The collective results confirm the presence of both the TPT and ER catalysts are necessitated within the same singlechain polymer system and importantly, the improved yields and acceleration in rate is, indeed, a result of polymer confinement and subsequent co-localization of TPT and the pyrene ER within the nanoreactors.

Table 1: Comparison of monocatalytic polymer systems with small-molecule ER or TPT in the [2+2]dimerization of anethole.

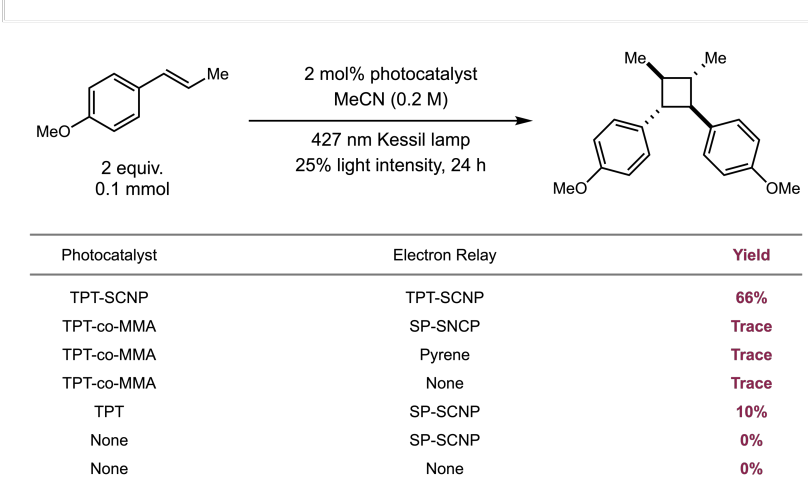

Upon seeing the effects of dual catalyst confinement in the dimerization of anethole, we looked to additional styrenyl derivatives for [2+2] cycloaddition. Dimerization of 4-methoxystyrene was also possible up in $26 \%$ yield. In this case, competition with the formation of poly(4-methoxy)styrene occurred at room temperature, given methoxystyrene's reported cationic polymerization using TPT. ${ }^{34}$ Additionally, $\alpha$-asarone was cyclized to give the natural product $( \pm$ ) Magnosalin in $49 \%$ yield. More challenging electron-deficient vinyl arenes did not undergo the desired transformation.
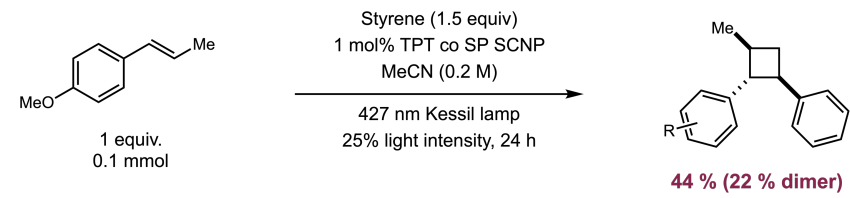

Figure 3: [2+2] Cross-dimerization of anethole with styrene.

We sought to further investigate the versatility and compatibility of our SCNP with cross [2+2] cycloadditions. (Figure 3). The cyclization of trans-anethole and styrene was used as a model system. Optimal conditions for the [2+2] cross-cycloaddition were found using 1.5 equivalents of styrene (See SI). Using our co-catalytic SCNPs, the cross product was obtained in $44 \%$ yield with $22 \%$ of the dimer with $1 \mathrm{~mol} \%$ SCNP with respect to TPT. Similar controls were conducted using the $[2+2]$ cross-dimerization system to probe the effects of confinement (Table 2).

Using TPT-co-MMA without any electron relay catalyst resulted in $5 \%$ of the desired product, with $3 \%$ of the trans-anethole dimer as the major byproduct. The addition of pyrene to this system resulted in trace amounts of the cross product and of the dimer. SP-co-MMA with TPT as a co-catalyst gave the cross-dimerized product in $1 \%$ yield with $2 \%$ of the dimer. The separate polymers SP-SCNP and TPT-co-MMA as co-catalysts afforded $5 \%$ of the desired product and 3\% of the byproduct, further highlighting the diffusion limitations present with monocatalytic polymers. 
Table 2: Comparison of monocatalytic polymer systems with small-molecule ER or TPT in [2+2]cross-dimerizations with anethole.

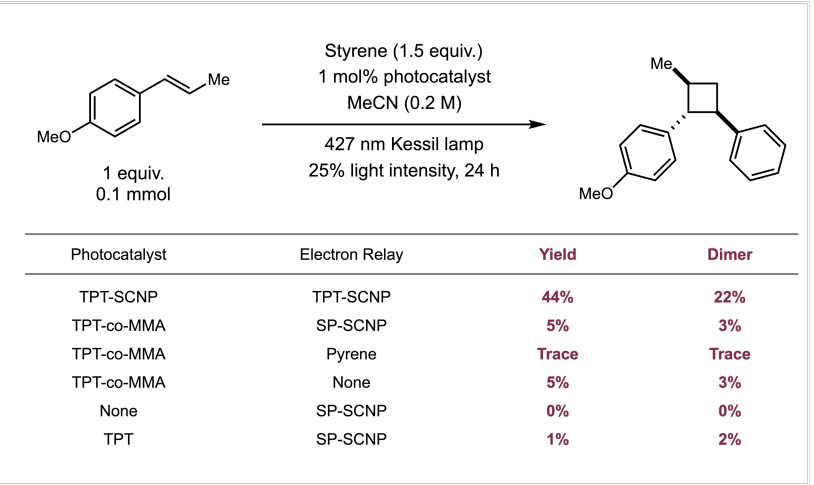

We demonstrate that a dual-catalytic single-chain polymer system bearing both photocatalyst and electron relay catalyst functions as an efficient homogeneous photoredox catalyst under confinement. Our investigations have highlighted that the proximity of the catalysts improves the efficiency of the co-catalyzed [2+2] cycloaddition compared with the analogous reaction using separate polymeric catalytic components. The enhanced reactions are attributed to improved efficiency of SET between the photocatalyst and the electron relay within the compartmentalized SCNP. The versatility of the photoredox SCNP-catalyst was demonstrated through both cross-dimerizations with styrene, and the synthesis of the natural product $( \pm)$ Magnosalin using low catalyst loadings. Future work will look interrogate other organo-photoredox catalysis with efforts toward tackling diffusion limitations.

\section{ASSOCIATED CONTENT}

\section{Supporting Information}

Small-molecule and polymer experimental procedures, spectroscopic characterization, optimization tables (PDF)

\section{AUTHOR INFORMATION}

\section{Corresponding Author}

* elizabeth.elacqua@psu.edu

\section{Author Contributions}

The manuscript was written through contributions of all authors.

\section{ACKNOWLEDGMENT}

This work was supported by start-up funds generously provided by the Pennsylvania State University. We acknowledge Joey Cotruvo and Christian Pester for the use of instruments, and we thank Eric Nacsa for helpful discussions.

\section{REFERENCES}

1. Mouarrawis, V.; Plessius, R.; van der Vlugt, J. I.; Reek, J. N. H., Front. Chem. 2018, 6 (623).

2. Kang, J.; Rebek, J., Nature 1997, 385 (6611), 50-52.

3. Caulder, D. L.; Powers, R. E.; Parac, T. N.; Raymond, K. N., Angew. Chem. Int. Ed. 1998, 37 (13-14), 1840-1843.

4. Dhakshinamoorthy, A.; Li, Z.; Garcia, H., Chem. Soc. Rev. 2018, 47 (22), 8134-8172.
5. Romero, N. A.; Nicewicz, D. A., Chem. Rev. 2016, 116 (17), 10075-10166.

6. Feng, Z.; Zeng, T.; Xuan, J.; Liu, Y.; Lu, L.; Xiao, W. J., Sci. China: Chem. 2016, 59, 171.

7. Skubi, K. L.; Blum, T. R.; Yoon, T. P., Chem. Rev. 2016, 116 (17), 10035-10074.

8. Kalyani, D.; McMurtrey, K. B.; Neufeldt, S. R.; Sanford, M. S., J. Am. Chem. Soc. 2011, 133 (46), 18566-18569.

9. Ye, Y.; Sanford, M. S., J. Am. Chem. Soc. 2012, 134 (22), 9034-9037.

10. Reed, N. L.; Herman, M. I.; Miltchev, V. P.; Yoon, T. P., Org. Lett. 2018, 20 (22), 7345-7350.

11. Hossain, A.; Bhattacharyya, A.; Reiser, O., Science 2019, 364 (6439), eaav9713.

12. Noble, A.; McCarver, S. J.; MacMillan, D. W. C., J. Am. Chem. Soc. 2015, 137 (2), 624-627.

13. Nicewicz, D. A.; MacMillan, D. W. C., Science 2008, 322 (5898), 77.

14. Zuo, Z.; Ahneman, D. T.; Chu, L.; Terrett, J. A.; Doyle, A. G.; MacMillan, D. W. C., Science 2014, 345 (6195), 437.

15. Jouffroy, M.; Primer, D. N.; Molander, G. A., J. Am. Chem. Soc. 2016, 138 (2), 475-478.

16. Gutierrez, O.; Tellis, J. C.; Primer, D. N.; Molander, G. A.; Kozlowski, M. C., J. Am. Chem. Soc. 2015, 137 (15), 4896-4899. 17. Tellis, J. C.; Primer, D. N.; Molander, G. A., Science 2014, 345 (6195), 433.

18. Sherbrook, E. M.; Jung, H.; Cho, D.; Baik, M.-H.; Yoon, T. P., Chem.Sci. 2020, 11 (3), 856-861.

19. Daub, M. E.; Jung, H.; Lee, B. J.; Won, J.; Baik, M.-H.; Yoon, T. P., J. Am. Chem. Soc. 2019, 141 (24), 9543-9547. 20. Yoon, T. P., Acc. Chem. Res. 2016, 49 (10), 2307-2315.

21. Riener, M.; Nicewicz, D. A., Chem. Sci. 2013, 4 (6), 26252629.

22. Jespersen, D.; Keen, B.; Day, J. I.; Singh, A.; Briles, J.; Mullins, D.; Weaver, J. D., Org. Process Res. Dev. 2019, 23 (5), 1087-1095.

23. Caputo, J. A.; Frenette, L. C.; Zhao, N.; Sowers, K. L.; Krauss, T. D.; Weix, D. J., J. Am. Chem.Soc. 2017, 139 (12), 4250-4253.

24. Zhu, Y.-Y.; Lan, G.; Fan, Y.; Veroneau, S. S.; Song, Y.; Micheroni, D.; Lin, W., Angew. Chem. Int. Ed. 2018, 57 (43), 14090-14094.

25. Rothfuss, H.; Knöfel, N. D.; Roesky, P. W.; BarnerKowollik, C., J. Am. Chem. Soc. 2018, 140 (18), 5875-5881. 26. Frisch, H.; Menzel, J. P.; Bloesser, F. R.; Marschner, D. E.; Mundsinger, K.; Barner-Kowollik, C., J. Am. Chem. Soc. 2018, 140 (30), 9551-9557.

27. Knöfel, N. D.; Rothfuss, H.; Willenbacher, J.; BarnerKowollik, C.; Roesky, P. W., Angew. Chem. Int . Ed. 2017, 56 (18), 4950-4954.

28. Chen, J.; Wang, J.; Li, K.; Wang, Y.; Gruebele, M.; Ferguson, A. L.; Zimmerman, S. C., J. Am. Chem. Soc. 2019, 141 (24), 9693-9700.

29. Ischay, M. A.; Ament, M. S.; Yoon, T. P., Chem. Sci. 2012, 3 (9), 2807-2811.

30. Ischay, M. A.; Lu, Z.; Yoon, T. P., J. Am. Chem. Soc. 2010, $132(25), 8572-8574$.

31. García-Acosta, B.; García, F.; García, J. M.; Martínez-

Máñez, R.; Sancenón, F.; San-José, N.; Soto, J., Org. Lett. 2007, 9 (13), 2429-2432.

32. Kawata, H.; Niizuma, S., Bull. Chem. Soc., Jpn. 1989, 62 (7), 2279-2283.

33. Conrow, K.; Radlick, P. C., J. Org. Chem. 1961, 26 (7), 2260-2263.

34. Perkowski, A. J.; You, W.; Nicewicz, D. A., J. Am. Chem. Soc. 2015, 137 (24), 7580-7583. 
Table of Contents Image

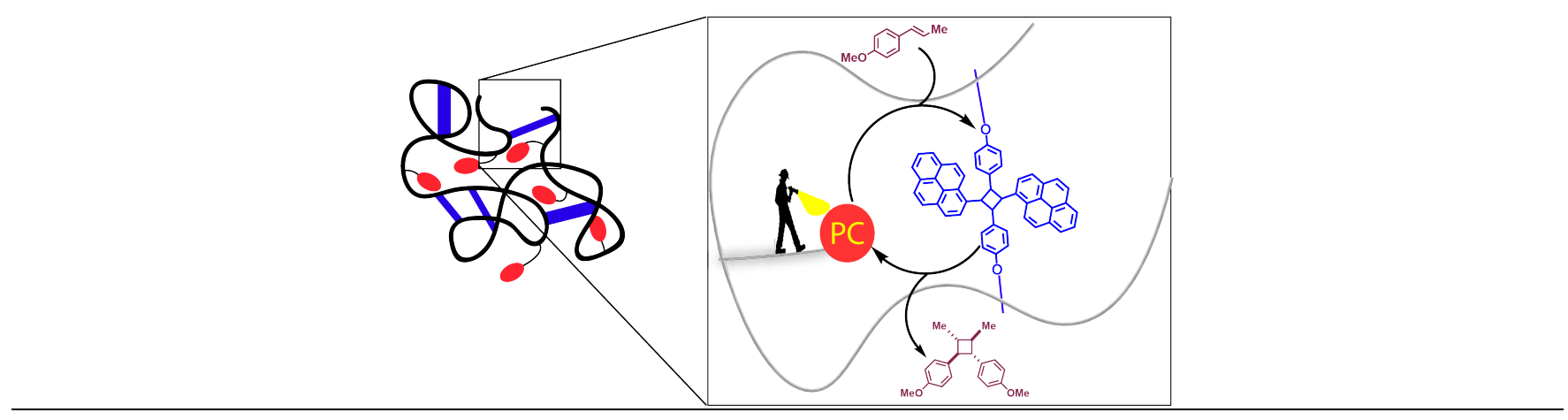

